\title{
A DYNAMICAL SURVEY OF INNER SOLAR SYSTEM ASTEROIDS
}

\author{
MARC A. MURISON \\ Astronomical Applications Dept., U.S. Naval Observatory, \\ 3450 Massachusetts Ave NW, Washington DC USA
}

\begin{abstract}
Results from a numerical integration survey of all 179 currently-known inner solar system asteroids with $a \leq a_{M a r s}, q \geq a_{M e r c u r y}$ are presented. A surprising number of asteroids are currently in, or very near, mean-motion resonances with Mercury, Venus, Earth, or Mars. Some of the resonance associations are of high order. Most of the resonance associations are relatively short-lived, with the asteroids wandering in and out of resonance on timescales of hundreds to several thousand years.
\end{abstract}

Integrations and analyses were performed with Newton, a solar system numerical integration computer program that is able to automatically identify mean-motion resonances of any order (http://aa.usno.navy.mil/Newton/). Initial conditions for the planets were taken from DE405. The integration method used in this study is a variable step size Bulirsch-Stoer. All planets - Mercury through Neptune were included in the purely gravitational force calculations. Earth and Moon were treated as one body.

Asteroid initial conditions were obtained from the Lowell Obs. list and recent MPC Circulars. In total, there were 179 asteroids that satisfied the selection criteria $a \leq a_{M a r s}, q \geq a_{\text {Mercury }}$. The latter condition was imposed in order to exclude the asteroids that would potentially require general relativistic corrections.

Four stages of resonant asteroid filtering were employed. The initial integration was for 500 Earth years. Asteroids that appeared to be in, or could not be considered excluded from, one or more mean-motion resonances with any of the planets Mercury through Saturn were then integrated for 1000 years. Similarly, survivors went on to 3000 years, then to 10,000 years. An asteroid is here considered not excludable from a $(p+q): p$ mean-motion resonance if the critical angle does not circulate within the integration time span.

The algorithm employed by Newton does not actually detect libration per se of the critical angle, but rather it detects circulation. Possible libration is inferred from a demonstrated lack of circulation over some interval. Hence, the algorithm
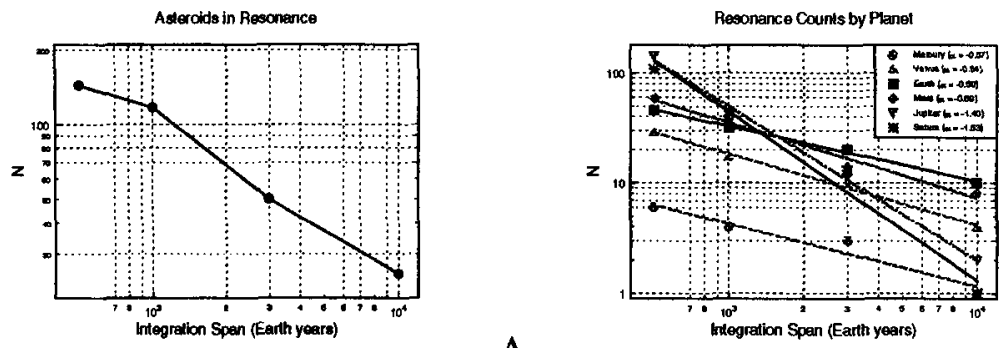

A.

B.

Fig. 1. A. Number of asteroids not classifiable as not being in resonance as a function of integration time. B. Resonance counts per planet as a function of integration time. 


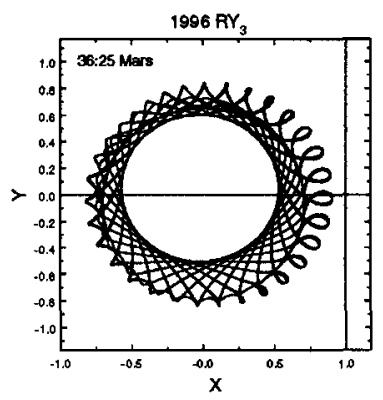

A.
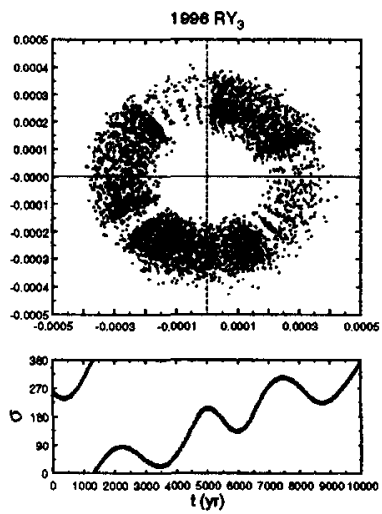

B.

Fig. 2. A. 500-year orbit trace of $1996 R Y_{3}$ projected onto the rotating xy plane. Mars is fixed at $(1,0)$. B. The resonance critical angle for $1996 R Y_{3}$ for 10,000 years.

cannot say for certain whether or not an asteroid is in a mean-motion resonance, but it can conclusively tell if an asteroid is not.

Figures 1 show the main results. The number of asteroids that are not excludable from being in resonance, as a function of integration time, is shown in Fig. 1A. The decline is fit well by a power law $N=N_{0} t^{\alpha}$ with slope $\alpha=-0.58$ and $N_{0}=5250$. In Fig. 1B (note the ordinate scale change) we have, for each planet separately, the number of mean-motion resonances associated with that planet as a function of integration time. Again, we see a power law dependence, but with two groupings. Resonance counts associated with the inner planets Mercury through Mars exhibit one behavior, with slopes in the range $-0.7<\alpha<-0.5$, while those of the two outer planets Jupiter and Saturn behave differently, their slopes being much larger, $\alpha \approx-1.5$. Due to multiple resonances for some asteroids, resulting from "slow drifters" contamination (see below), there appear to be more resonance associations (Fig. 1B) than there are asteroids in resonance (Fig. 1A). The outer planet grouping turns out to consist almost exclusively of slow drifters. As the integration time increases, more and more of these are shown to be circulating in the critical angle. By $t=10,000 \mathrm{yr}$, all but a few have disappeared. Contamination for the inner planets is much less severe, since the planetary periods are shorter, leading to quicker identification of circulators.

Figure $2 \mathrm{~A}$ shows a 500-year orbit trace of a resonating asteroid projected onto the orbital plane of the reference planet. Figure 2B shows the corresponding critical angle for an integration covering a span of 10,000 years. The radius in the upper panel is $\mathbf{r}=\left[\left(a-a_{\min }\right)+\frac{1}{2}\left(a_{\max }-a_{\min }\right)\right] \cdot(\cos \sigma, \sin \sigma)$

$1996 R Y_{3}$ is an example of a "slow drifter". It is very near the $36: 25$ resonance with Mars, and it is strongly influenced by the close proximity of that resonance, but the critical angle clearly circulates. 